\title{
The Association Between HSP90/topoisomerase I Immunophenotype and the Clinical Features of Colorectal Cancers in Respect to KRAS Gene Status
}

\author{
JULIA K. BAR ${ }^{1}$, ANNA LIS-NAWARA ${ }^{1}$, PIOTR GRELEWSKI ${ }^{1}$, LESZEK NOGA ${ }^{2}$, \\ ZYGMUNT GRZEBIENIAK ${ }^{3}$ and MICHAŁ JELEN' ${ }^{4}$ \\ ${ }^{1}$ Department of Immunopathology and Molecular Biology, ${ }^{2}$ Department of Pathophysiology, \\ ${ }^{3}$ Second Department and Clinic of General and Oncological Surgery, \\ ${ }^{4}$ Department of Pathomorphology Wroclaw Medical University, Wroclaw, Poland
}

\begin{abstract}
Aim: The aim of this study was to investigate heat shock protein 90 (HSP90) and topoisomerase I (Topo I) expression and the association between both proteins and clinicopathological parameters of colorectal cancer (CRC), in order to describe their role in tumor biology regarding to Kirsten Ras (KRAS) - positive/negative cases. Materials and Methods: Expression of HSP90 and Topo I, and KRAS gene mutations were estimated in primary CRCs. Results: HSP90/Topo I immunophenotype correlated with gender, Duke staging, tumor grade and lymph node metastasis $(p<0.01)$. Positive correlation was found between KRAS mutation and HSP90 expression ( $p=0.02)$. HSP90, Topo I expression, and co-expression of HSP90/Topo I correlated with unfavorable parameters of CRCs in respect to KRAS gene status $(p<0.001)$. Conclusion: Our results revealed that cooperation between HSP90 and Topo I expression exists in CRCs, independently of KRAS gene status, suggesting that co-expression of both proteins might be considered as a double target on individual tumor cells.
\end{abstract}

Colorectal cancer (CRC) is one of the most biologicallyaggressive types of cancer $(1,2)$. The five-year survival rate of patients with metastatic disease is as low as $10-20 \%$ (3, 4). The identification of specific molecules expressed by cancer cells as prognostic and predictive biomarkers is very important in order to describe the phenotypes of patients with CRC who respond to therapy $(5,6)$. Up to now, in

Correspondence to: Julia K. Bar, Department of Immunopathology and Molecular Biology, University Medical Wroclaw, Borowska 213, 50-556 Wrocław, Poland. Tel: +48 717343955, Fax: +48 717343968,e-mail: julia.bar@umed.wroc.pl

Key Words: Colorectal cancer, HSP90, Topo I expression, KRAS mutation. advanced CRC, the KRAS gene is one of the most important predictive biomarkers for response to anti-epidermal growth factor receptor (EGFR)-targeted therapy $(1,4,7)$.

Detection of KRAS mutations is related with reduced response to anti-EGFR agents and the benefits of treatment with anti-EGFR antibody in patients with advanced stage of the disease are restricted to the group with KRAS wild-type gene $(8,9)$. Clinical trials showed that the outcome of patients with wild-type KRAS was significantly better than patients with KRAS mutation $(8,10,11)$. Based on KRAS gene status, CRCs are divided into two different subgroups with a different clinical outcome $(4,7,10,11)$. Taking into account that tumors are not monoclonal but biologically heterogeneous, recent data consider the concept of personalized cancer therapy in solid tumors $(10,11)$. In KRAS- positive/negative CRC cases, personalized treatment strategy is focused on the identification of more than one biomarkers on tumor cells, an approach that might allow the prediction of the response of CSCs to the offered regimens (4). So, it is important to describe the correlation between KRAS status and other biological biomarkers, in order to find out a potential new treatment target in tumor cells. Several biomarkers have been analyzed in CRCs, but only a few have clinical application $(4,7)$.

Human DNA topoisomerases are essential nuclear enzymes that make structural changes in the topological state of DNA without changing the primary structure. Type I topoisomerase (Topo I) introduces a transient single-strand break into the double-stranded DNA, passes the DNA strands through one another and religates the break $(12,13)$. Topo I is the target for the camptothecin derivatives, irinotecan and topotecan, and some data show that higher levels of Topo I expression predict response to irinotecan, in CRC patients $(2,12)$. There is increasing evidence for the beneficial use of Topo I inhibitors in cancer clinical trials $(12,14)$. Observations reviewed by Alagoz et al. suggest that the combination of irinotecan with radiotherapy might increase 
the range of killed tumor cells (15). There are also reports on targeted therapy against Topo I and other biomarkers located on tumor cells in individual patients $(7,12)$.

The second candidate of the new, innovative molecular therapy of CRC is heat shock protein 90 (HSP90) $(6,7)$. HSP90 is an important molecular chaperone for protein folding, transmission, and intracellular localization, in both normal and tumor cells $(16,17)$. HSP90 is an important regulator of cell growth and survival, as well as being essential for the activity of a wide range of key oncogenic proteins, including kinases, transcription factors, and suppressor proteins $(17,18)$. Many HSP90 client proteins are involved in processes such as proliferation, apoptosis, and cycle progression $(16,18)$. Several reports have analyzed HSP90 expression in solid tumors and found that HSP90 expression is correlated with clinical prognosis, $(5,16,19$, 20). Xu et al. (16) and Ahn et al. (21) demonstrated that high HSP90 protein expression is related to the metastasis and invasion of CRC. Preclinical studies and early clinical trials have confirmed the efficacy of HSP90 inhibition as a target therapy for many solid tumors $(6,16,19,20)$. Recent data showed the therapeutic utility of HSP90 inhibitors in patients resistant to tyrosine kinase inhibitors $(7,18)$. Little is known about the role of Topo I and HSP90 protein expression in KRAS-positive and negative CRCs, as well as the relationship between Topo I and HSP90 in CRC is not clear.

The aim of the study was to investigate Topo I and HSP90 expression and the association between both proteins and clinicopathological parameters of CRC, in order to describe the role of Topo I and HSP90 expression in the biological behavior of tumor cells, regarding to the KRAS gene status of CRC.

\section{Materials and Methods}

Patients. The study was performed on formalin-fixed and paraffinembedded (FFPE) archival cancer tissues. One hundred patients with primary $\mathrm{CRC}$, before therapy, were included in the study. All patients underwent surgery between December 2009 and July 2015 , in the Second Department and Clinic of General and Oncological Surgery of Medical University, Wroclaw, Poland. The patient cohort consisted of 51 male and 49 female individuals, the median age was $62.4(38-82)$ years, and assessment of the clinical stage of disease revealed 15 cases at Dukes A, 37 cases at Dukes B, and 48 cases at Dukes C.

Tissue specimens. One hundred CRCs included 58 cases with colon cancer and 42 with rectum cancer. Tumor specimens from CRCs were histologically verified by two independent pathologists to confirm the histological type and grade, according to the current World Health Organization criteria (22). The study group comprised of 89 papillary or tubular adenocarcinoma, 9 mucinous adenocarcinomas and 2 signet ring cell adenocarcinomas. Thirteen tumors were well, 65 moderately, and 22 poorly differentiated; 48 cases were with lymph node metastasis and 52 without lymphatic metastasis.
DNA extraction. Sixty seven from the 100 CRC cancer cases were classified for DNA extraction. Serial 5- $\mu$ m sections were taken from FFPE tissues. The last section was stained with hematoxilin-eosin (H\&E), the tumor area was marked, and the percentage of tumor cells was estimated by a pathologist. A material with $>40 \%$ tumor content by area was used for further procedure. The tumor tissue was submitted to xylene deparaffinization and was incubated for $1 \mathrm{~h}$ at $56^{\circ} \mathrm{C}$ in $180 \mu \mathrm{l}$ of ATL buffer and $20 \mu \mathrm{l}$ of proteinase K. DNA was purified using the QIAamp DNA, FFPE Tissue Kit, QIAGEN (Qiagen, Hilden, Germany) and finally reconstituted in $50 \mu$ of kitprovided ATE buffer. DNA content was measured with a spectrophotometer (MaestroNano Micro-Volume Spectrophotometer, Maestrogen, Ramsey, MN, USA) and was kept at $4^{\circ} \mathrm{C}$ before use.

KRAS mutation analysis. DNA extracted from FFPE tumor samples was used for KRAS gene status analysis. The mutation status of KRAS exon 2 at codon 12 and 13 was estimated by the TheraScreen ${ }^{\circledR}$ KRAS RGQ PCR Kit (CE-IVD) (Qiagen Manchester Ltd, Manchester, UK), which combines two technologies (ARMS and Scorpions) to detect the most commonly reported KRAS mutations by RT-PCR. Total sample volume was at $25 \mu \mathrm{l}$. The reaction conditions were as follows: $95^{\circ} \mathrm{C}$ for $15 \mathrm{~min}$ to activate the Taq polymerase, 40 cycles of $95^{\circ} \mathrm{C}$ for $30 \mathrm{~s}$ to denature, $60^{\circ} \mathrm{C}$ for $60 \mathrm{~s}$ to anneal and extend. RT-PCR was performed with a Rotor Gene Q 5000 (Qiagen, New South Wales, Australia).

DNA samples were screened for the following mutations: p.Glyc12Ser (c.34G $>$ A), p.Gly12Arg (c.34G $>$ C), p.Gly12Cys (c.34G>T), p.Glyc12Asp (c.35G>A), p.Gly12Ala (c.35G>C), p.Gly12Val (c.35G>T), p.Gly13Asp (c.38G>A).

Immunohistochemistry (IHC). Immunohistochemical staining for protein analysis was performed on FFPE tissue, using the Universal Dako REAL EnVision Detection System, Peroxidase/DAB+, Rabbit/Mouse (Dako, Copenhagen, Denmark) for visualization of the primary monoclonal antibodies: anti-Topo I that binds to a region within the middle of the Topo I molecule (clone 1D6, Novocastra, Newcastle, UK) and anti-HSP90 protein that recognizes protein corresponding to 306 amino acids of the $\mathrm{C}$ terminus of the HSP90 molecule (clone JPB24, Novocastra, Newcastle, UK). Five- $\mu \mathrm{m}$ sections from one selected block from each tumor were deparaffinized and boiled 3 times for 5 min for each antibody in citrate buffer $(\mathrm{pH}=6.0)$ at $700 \mathrm{~W}$ in a microwave. After the microwave treatment, the tissue sections were slowly cooled for $20 \mathrm{~min}$. Nonspecific tissue and endogenous peroxidase reactivity were blocked with Dako REAL Peroxidase Blocking Solution (Dako, Copenhagen, Denmark). Tissue specimens were incubated with primary antibodies (anti-Topo I, 1:50 dilution and anti-HSP90 protein, 1:400 dilution) overnight at $4^{\circ} \mathrm{C}$. Following washing with $0.1 \mathrm{M}$ Tris-buffer, $\mathrm{pH}=7.4$ (TBS), the tissue specimens were incubated with Dako REAL EnVision/HRP, Rabbit/Mouse (Dako, Copenhagen, Denmark) for $30 \mathrm{~min}$ at room temperature. After washing with TBS, the antigen-antibody reaction was visualized by DAB (3,3 diaminobenzidine) chromogen (Dako, Denmark) (4 min incubation at room temperature). Sections were counterstained with hematoxylin and mounted on slides Samples incubated with buffer (TBS) without primary antibodies were used as negative control.

Interpretation of immunochistochemical staining. Specimens were scored semiquantitatively, taking into account the intensity of 
Table I. Relationship between heat shock protein 90 (HSP90), topoisomerase I (Topo I) and HSP90/Topo I expression and the clinicopathological features of colorectal cancer.

\begin{tabular}{|c|c|c|c|c|c|c|c|}
\hline \multirow[t]{3}{*}{ Parameters } & \multicolumn{6}{|c|}{ Immunopositivity ( $\%$ of positive cases) } & \multirow[b]{3}{*}{$p$-Value } \\
\hline & \multicolumn{2}{|c|}{ HSP90 } & \multicolumn{2}{|c|}{ Topo I } & \multicolumn{2}{|c|}{ HSP90/Topo I } & \\
\hline & $\mathrm{n}$ & Positive (\%) & $p$-Value & Positive (\%) & $p$-Value & Positive (\%) & \\
\hline \multicolumn{8}{|c|}{ Age (at surgery) } \\
\hline$<65$ years & 46 & $27(58.7)$ & NS & $21(45.6)$ & NS & $16(34.8)$ & NS \\
\hline$>65$ years & 54 & $38(70.4)$ & & $26(48.1)$ & & $25(46.3)$ & \\
\hline \multicolumn{8}{|l|}{ Gender } \\
\hline Male & 51 & $29(56.9)$ & NS & $21(41.2)$ & NS & $16(31.4)$ & 0.04 \\
\hline Female & 49 & $36(73.5)$ & & $26(53.0)$ & & $25(51.0)$ & \\
\hline \multicolumn{8}{|l|}{ Tumor site } \\
\hline Colon & 58 & 45 (77.6) & 0.001 & $30(51.7)$ & NS & $28(48.3)$ & NS \\
\hline Rectum & 42 & $20(47.6)$ & & $17(40.5)$ & & $13(30.9)$ & \\
\hline \multicolumn{8}{|c|}{ Histological grade } \\
\hline G1 & 13 & $6(46.2)$ & & $3(23.0)$ & & $3(23.0)$ & \\
\hline G2 & 65 & $41(63.0)$ & NS & $26(40.0)$ & 0.005 & $21(32.3)$ & 0.0003 \\
\hline G3 & 22 & $18(81.8)$ & & $18(81.8)$ & & $17(77.3)$ & \\
\hline \multicolumn{8}{|l|}{ Stage } \\
\hline Duke A & 15 & $5(33.3)$ & & $3(20.0)$ & & $3(20.0)$ & \\
\hline Duke B & 37 & $20(54.0)$ & 0.0004 & $10(27.0)$ & 0.00002 & $8(21.6)$ & 0.0001 \\
\hline Duke C & 48 & $40(83.3)$ & & $34(70.8)$ & & $30(62.5)$ & \\
\hline \multicolumn{8}{|c|}{ N Classification } \\
\hline No & 52 & $22(42.3)$ & 0.00001 & $15(28.9)$ & 0.0001 & $11(21.2)$ & 0.00003 \\
\hline $\mathrm{N}+$ & 48 & 43 (89.6) & & $32(66.7)$ & & $30(62.5)$ & \\
\hline
\end{tabular}

The significance of data is shown in bold, NS: non statistically significant.

immunostaining and the number of tumor cells exhibiting staining for Topo I and HSP90 proteins.

Expression of HSP90 was assessed by determination of cytoplasmic staining based on the intensity of immunostaining and the percentage of stained tumor cells. The percentage of Topo I-positive cells were determined by counting 1000 cells in randomly selected 10-highpower fields, by two independent observers, using a double-headed BX51 Olympus microscope (Olympus, Toyo, Japan). A ratio of positive to total cells less than $20 \%$, for both proteins, was interpreted as negative. Positive cells proportion and staining intensity were considered in the scoring system. The percentage of HSP90 and Topo I positive tumor cells was scored as follows: $0=0-20 \%, 1=21-40 \%$, $2=41-70 \%, 3=71-100 \%$. The scoring criteria for staining intensity were as follows: $0=$ no immunostaining, $1=$ =weak (light yellow), $2=$ moderate (medium brown) and 3 =strong (brown). The overall estimation (the sum of the two scores) was classified into two categories, as high or low immunostaining. For all statistical analyses, HSP90 and Topo I expression was divided into two groups: negative or limited to $20 \%$ of positive tumor cells versus $>20-100 \%$ of positive cells.

Statistical analysis. Associations between HSP90 and Topo I expression, HSP90/Topo I co-expression, KRAS mutation and clinicopathological parameters of CRCs were statistically studied by Chi-square test. Association between HSP90 and Topo I expression as well as between HSP90, Topo I expression and KRAS gene status was analyzed by Spearman rank correlation. Comparative analysis of studied proteins in relation to clinicopathological parameters between KRAS-positive versus KRAS-negative CRCs was performed by Wilcoxon signed rank test. Differences were considered as statistically significant when $p \leq 0.05$.

\section{Results}

Association of HSP9O and Topo I expression with clinicopathological parameters of CRC. Overexpression of HSP90 protein was found in $65(65.0 \%)$ of the 100 CRCs. HSP90 positive tumor cells were dominated in glandular structures of tumor tissue (Figure 1). An association between HSP90 protein expression and Duke staging ( $p=0.0004)$, presence of lymph node metastasis $(p=0.00001)$, and tumor site of CRC was found ( $p=0.001)$ (Table I).

Topo I expression was observed in 47 (47.0\%) of the 100 CRCs. Topo I-positive cells were distributed throughout the tumor specimens and the expression of this enzyme was stronger in glandular tissue structure than in solid tissue of CRC (Figure 2). The Topo I-positive cases occurred more frequently in Duke C $(p=0.00002)$, high tumor grade $(p=0.005)$, and in CRCs with presence of lymph node metastasis $(p=0.0001)$ (Table I). There was a positive correlation between HSP90 and Topo I expression in the analyzed group of CRCs $(p=0.00001)$. 


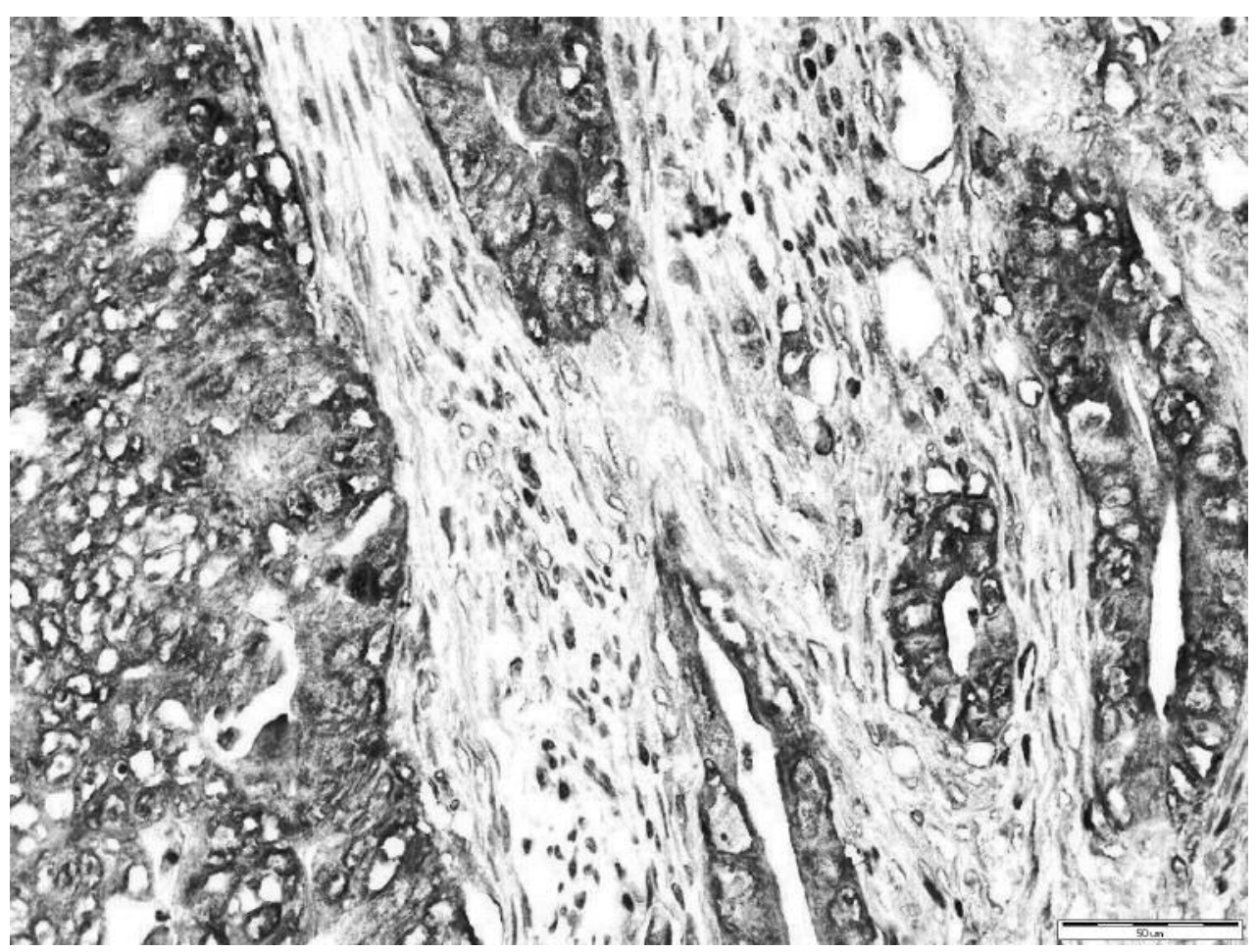

Figure 1. Strong cytoplasmic expression of heat shock protein 90 (HSP90) in colorectal cancer. Heterogenous pattern of HSP90 immunostaining is visible (EnVision technique). Scale bar $=50 \mu \mathrm{m}$.

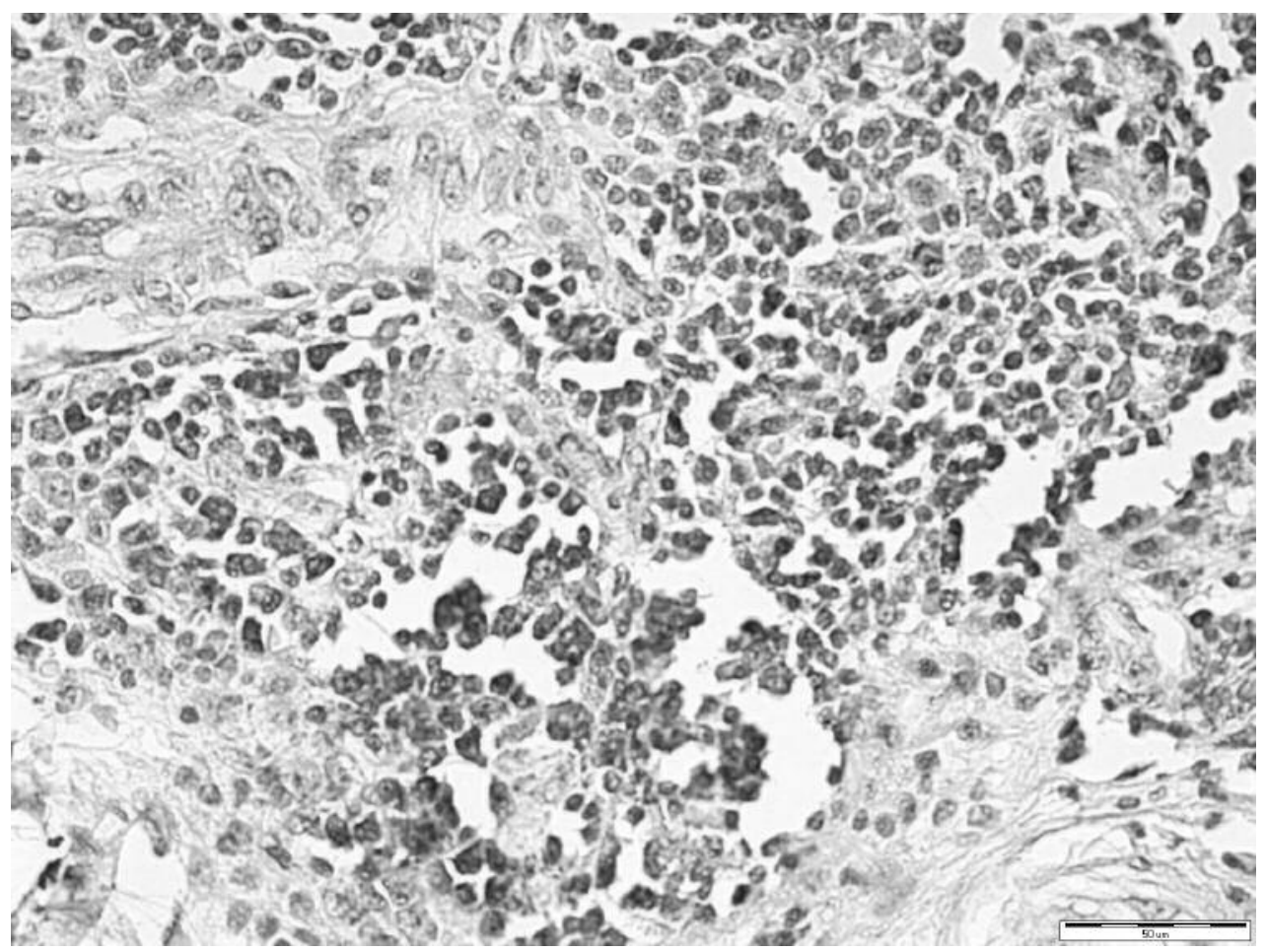

Figure 2. Immunohistochemical staining for topoisomerase I (Topo I) enzyme in colorectal cancer. Nuclear accumulation of Topo I was observed in high percentage of tumor cells (EnVision technique). Scale bar=50 $\mu \mathrm{m}$. 


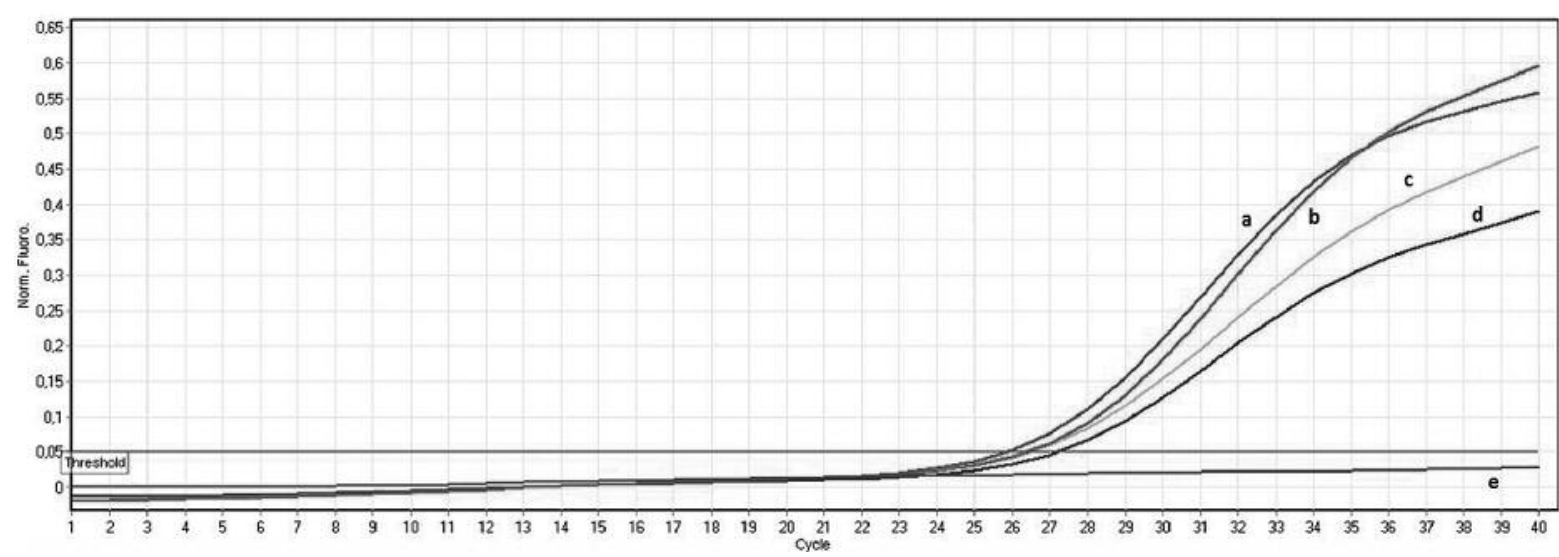

Figure 3. Kirsten Ras (KRAS) mutation in colorectal cancer. Two mutations of KRAS gene were frequently observed in our study: a - positive control, $b$ - control DNA, c - mutation c.35 GGT>GAT, $d$ - mutation c.38 GGC>GAC, e - negative control.

The HSP90/Topo I immunophenotype was associated with high tumor grade $(p=0.0003)$, presence of lymph node metastasis $(p=0.00003)$, advanced stage of disease $(p=0.0001)$ and female patients with CRC $(p=0.04)$.

KRAS mutations in CRCs. KRAS mutations were detected in $19(28 \%)$ of 67 CRCs. KRAS mutations were observed more often in tumor showing lymph node metastasis $(p=0.04)$ (Table II). Nineteen different types of mutations in exon 2 , codons 12 and 13, found in the analyzed cases are presented in Table III. The most prevalent of KRAS mutations were located in codon 12 , specifically c.35 GGT $>$ GAT $(38.0 \%)$, and codon 13 c.38 GGC>GAC (28.0\%) (Figure 3). Double mutation was found in two patients; one patient had c.34

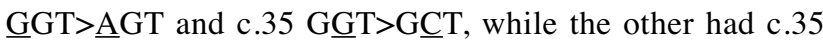
GGT $>$ GCT and c. 35 GGC $>$ G $\underline{A C}$.

HSP90 and Topo I expression was analyzed for KRAS gene status in CRCs. The positive correlation between KRAS mutation and high HSP90 protein expression $(p=0.02)$, but not between KRAS and Topo I nor Topo I and HSP90 expression was found in KRAS analyzed group of CRCs (data not shown). HSP90 and Topo I expression as well as parallel expression of both proteins was found more frequently in advanced stage of diseases $\left(p_{\mathrm{HSP} 90}=0.00001\right.$, $p_{\text {TopoI }}=0.00009$ and $\left.p_{\text {HSP90/TopoI }}=0.00008\right)$, high tumor grade $\left(p_{\mathrm{HSP}} 90=0.0002, p_{\text {TopoI }}=0.00001, p_{\mathrm{HSP}} 90 / \mathrm{TopoI}=\right.$ $0.000001)$, presence of lymph node metastasis $\left(p_{\mathrm{HSP}} 90=\right.$ $0.000001, p_{\text {TopoI }}=0.00002, p_{\text {HSP90 }} /$ TopoI $\left.=0.000001\right)$ and colon location $\left(p_{\mathrm{HSP}}=0.000001, p_{\text {TopoI }}=0.0004\right.$ and $p_{\text {HSP90/TopoI }}=0.00004$ ) (Table II). The association between HSP90/Topo I immunophenotype and clinicopathological parameters of CRC is presented in Figure 4. HSP90/Topo I immunophenotype and Topo I expression was associated with the female group $(p=0.038)$.

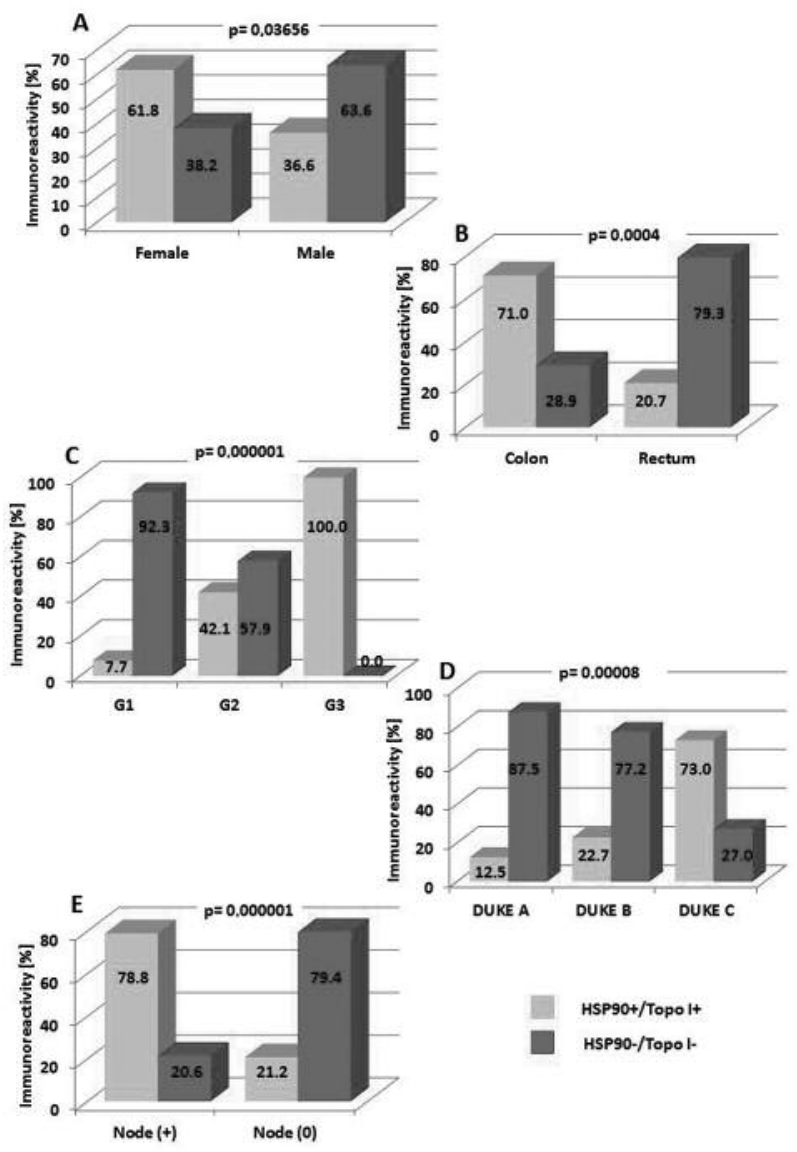

Figure 4. Correlation between heat shock protein/topoisomerase I (HSP90/Topo I) immunophenotype and clinicopathological parameters of colorectal cancers analysed for Kirsten Ras (KRAS) gene status. (A) HSP90/Topo I immunophenotype was associated with female group $(p=0.038),(B)$ with rectum tumor site $(p=0.0004),(C)$ with high tumor grade $(p=0.000001),(D)$ with advanced clinical stage of disease ( $p=0.00008),(E)$ with colorectal cancer presence of lymph node metastasis $(p=0.000001)$. 
Table II. Relationship between Heat shock protein 90 (HSP90), Topoisomerase I (Topo I) expression, Kirsten Ras (KRAS) mutation, HSP90/Topo I immunophenotype and the clinicopathological features of colorectal cancer.

\begin{tabular}{|c|c|c|c|c|c|c|c|c|c|}
\hline \multirow[t]{3}{*}{ Parameters } & \multicolumn{3}{|c|}{ Mutation } & \multicolumn{4}{|c|}{ Immunopositivity } & & \\
\hline & \multirow[b]{2}{*}{$\mathrm{n}$} & \multicolumn{2}{|c|}{ KRAS } & \multicolumn{2}{|c|}{ HSP90 } & \multicolumn{2}{|c|}{ Topo I } & \multicolumn{2}{|c|}{ HSP90/Topo I } \\
\hline & & Positive (\%) & $p$-Value & Positive (\%) & $p$-Value & Positive (\%) & $p$-Value & Positive (\%) & $p$-Value \\
\hline \multicolumn{10}{|c|}{ Age (at surgery) } \\
\hline$<65$ years & 31 & $8(25.8)$ & NS & $22(71.0)$ & NS & $14(45.2)$ & NS & $12(38.7)$ & NS \\
\hline$\geq 65$ years & 36 & $11(30.6)$ & & $25(69.4)$ & & $21(58.3)$ & & $21(58.3)$ & \\
\hline \multicolumn{10}{|l|}{ Gender } \\
\hline Female & 34 & $12(35.3)$ & NS & $25(73.5)$ & NS & $22(64.7)$ & 0.038 & $21(61.8)$ & 0.038 \\
\hline Male & 33 & $7(21.2)$ & & $22(66.7)$ & & $13(39.4)$ & & $12(36.6)$ & \\
\hline \multicolumn{10}{|l|}{ Tumor site } \\
\hline Colon & 38 & $14(36.8)$ & NS & $38(100.0)$ & 0.000001 & $27(71.0)$ & 0.0004 & $27(71.0)$ & 0.00004 \\
\hline rectum & 29 & $5(17.2)$ & & $9(31.0)$ & & $8(27.6)$ & & $6(20.7)$ & \\
\hline \multicolumn{10}{|c|}{ Histological grade } \\
\hline G1 & 13 & $3(23.1)$ & & $4(30.8)$ & & $1(7.7)$ & & $1(7.7)$ & \\
\hline $\mathrm{G} 2$ & 38 & $11(28.9)$ & NS & $27(71.0)$ & 0.0002 & $18(47.4)$ & 0.00001 & $16(42.1)$ & 0.000001 \\
\hline G3 & 16 & $5(31.3)$ & & $16(100.0)$ & & $16(100.0)$ & & $16(100.0)$ & \\
\hline \multicolumn{10}{|l|}{ Stage } \\
\hline Duke A & 8 & $0(00.0)$ & & $1(12.5)$ & & $1(12.5)$ & & $1(12.5)$ & \\
\hline Duke B & 22 & $6(27.3)$ & NS & $12(54.6)$ & 0.00001 & $6(27.3)$ & 0.00009 & $5(22.7)$ & 0.00008 \\
\hline Duke C & 40 & $13(35.1)$ & & 34 ( 91.9) & & $28(75.7)$ & & $27(73.0)$ & \\
\hline \multicolumn{10}{|c|}{ N Classification } \\
\hline No & 34 & $6(17.6)$ & 0.04 & $15(44.1)$ & 0.000001 & $9(26.5)$ & 0.00002 & 7 (20.6) & 0.000001 \\
\hline $\mathrm{N}+$ & 33 & $13(39.4)$ & & $32(97.0)$ & & $26(78.8)$ & & $26(78.8)$ & \\
\hline
\end{tabular}

The significance of data is shown in bold, NS: Non statistically significant.

\section{Discussion}

Our results concerning HSP90 protein expression and the percentage of positive cells in CRCs are in agreement with earlier published reports $(5,16)$. Different percentages of $\mathrm{HSP}^{+} 0^{+}$cells ranging from $20-90 \%$ that were observed between CRC samples in the present study, might be important for the interaction between HSP90 protein and other oncogenic molecules such as AKT, RAF, RAS and p53, determining individual features of CRCs that reflect their biological behavior (17). Based on previous data $(5,7)$ and our observation, we might conclude that the number of HSP90 positive cells is crucial for the efficiency of HSP90-targeted therapy in CRC. Our data and results of some reports, in which HSP90 expression was associated with advanced disease stage of disease, presence of lymph node metastasis, and tumor location in colon cancer, indicate that HSP90 might be also involved in CRC progression $(5,16)$. In an in vitro study, authors have found that blocking of the HSP90-Src-mediated pathway effectively suppresses tumor growth and metastasis of 5-fluorouracil (FU) resistant colon cancer and they suggest that targeting of HSP90 or Src could be used in clinical strategies to overcome 5-FU chemoresistance (21). Contrary to ours and others observations $(5,16)$, Drecoll et al. (6) suggest that high
Table III. KRAS mutations detected in individual colorectal cancers.

\begin{tabular}{lccccc}
\hline \multicolumn{5}{c}{ KRAS mutant } \\
\hline Exon & Codon & Mutation & $\begin{array}{c}\text { Amino } \\
\text { acid }\end{array}$ & $\begin{array}{c}\text { No. of } \\
\text { patients }\end{array}$ & $\%$ \\
& & & 3 & $14 \%$ \\
2 & \multirow{2}{*}{12} & c.34 GGT>AGT & Gly12Ser & 3 & 0 \\
& & c.34 GGT>CGT & Gly12Arg & 0 & 0 \\
& c.34 GGT>TGT & Gly12Cys & 0 & $38 \%$ \\
& c.35 GGT>GAT & Gly12Asp & 8 & $9,5 \%$ \\
& c.35 GGT>GCT & Gly12Ala & 2 & $9,5 \%$ \\
& c.35 GGT>GTT & Gly12Val & 2 & $28 \%$ \\
\hline
\end{tabular}

level of HSP90 expression observed in earlier tumor stage, and absence of lymph node metastasis in colon carcinoma might be results of discrepancies, in the level of HSP90 protein expression, between the studies that depend on posttranslational modification or protein turnover. Nevertheless, there is the opinion that targeting of the HSP90 molecule might be an alternative therapeutic approach for colon cancers characterized by high expression of HSP90 protein $(6,21)$.

Azzoni et al. (23) have found Topo I expression in a percentage of CRCs similar to our study. However, there are 
data showing Topo I expression in high percentage (77\%) of CRCs (12). The observed variability in Topo I expression might be due to the result the score systems used to estimate the immunostaining, the type of the antibodies, and the size of samples among studies (23). However, both studies underline that basal level of Topo I expression in tumor tissue might be crucial to response to Topo I-targeted therapies $(12,23)$. According to the published reports, only high Topo I expression in CRC, as well as in colon cancer, is correlated to increased irinotecan sensitivity $(2,24,25)$. In agreement with a previous report (24), our study showed significant correlation between Topo I expression and tumor stage, presence of lymph node metastasis, and tumor grade of CRCs. Our data indicated that Topo I expression characterizes the subgroup of CRCs with aggressive behavior. This observation could be supported by a previous study on non-small lung cancer, which suggests Topo I as a prognostic factor, as its expression was inversely correlated with patient survival (14). Therefore, it seems reasonable to assume that the association of Topo I expression with unfavorable clinical parameters of CRC observed in the current study, indicates that this biomarker characterizes the subgroup of tumors that possess biological aggressive behavior (25). We found a positive correlation between HSP90 and Topo I expression in CRC. HSP90/Topo $\mathrm{I}^{+}$immunophenotype was dominated in cases showing worse clinical parameters (high tumor grade and stage, presence of lymph node metastasis, tumor colon location). There are no previous data analyzing correlation between HSP90 and Topo I in CRC. Results of this study could be compared with studies of Xu et al. (16), that revealed a positive correlation between HSP90 and HIF- $1 \alpha$ expression in $\mathrm{CRC}$, showing that they might exert a synergistic effect on the occurrence, development, invasion and metastasis of CRC. In previous studies, the observed association between Topo I and cycloxygenase-2 (COX2) expression in CRC, as well as between Topo I and thymidylate synthase (TYMS), suggest a regulatory role of Topo I expression for COX2 in DNA synthesis (24). Another report has shown association between Topo I expression and $18 \mathrm{q}$ allelic loss of heterozygosity $(\mathrm{LOH})$, microsatellite stable phenotype, and normal expression of mismatch proteins hMLH1 and hMSH2, indicating that this type of CRC might have a more favorable response to Topo I inhibitors therapy (23). Our findings are also partly comparable with the study of Drecoll et al. (6), which demonstrates a correlation between HSP90 and Her2 expression in primary colon carcinoma with lymph node metastasis and with data from Wang et al. (18), which show that HSP90 protein expression positively correlates with MMP9 tissue level, implying higher invasive and metastasizing activity of gastric carcinoma cells with high HSP90 expression. Taken together, earlier published data $(1,5,6,16,23,24)$ and our observations, suggest that co-expression of HSP90 and Topo I might be a biological feature of CRC, responsible for promoting aggressive tumor growth. We might postulate that targeted therapy against HSP90/ Topo I molecules expressed on the tumor cells, could improve the effectiveness of CRC treatment. In the present study, the results concerning KRAS mutation in CRC are in agreement with literature $(9,10,11$, 26). The frequency of KRAS mutation in CRC with lymph node metastasis, noted in the current study, was also observed by other authors $(9,26)$. Up to now, several reports have analyzed the expression of Topo I, human epidermal growth factor receptor 2 (Her2), HSP90, hMLH1 and hMSH2 proteins in CRC taking into account $K R A S$ gene status. However, the role of these proteins in respect to the presence or absence of KRAS mutation in CSCs is still unclear $(7,8,9,27)$. Similarly to an individual report (7), we found positive correlation between HSP90 expression and KRAS mutation. It proved that HSP90 expression might be a useful biomarker for targeted therapy in KRAS-mutated cases, which are resistant to cetuximab treatment (4). These data are supported by the observation that inhibition of HSP90 expression by ganetespib, in combination with platinum, in KRAS-mutated CRC cell lines might have the most potential therapeutic benefit (7). The lack of correlation between Topo I expression and KRAS gene status, found in the present study, indicated that inhibitors of Topo I might be used independently of $K R A S$ gene status in CRC. Interestingly, our data showed that in KRAS analyzed group HSP90/Topo I immunophenotype was associated with unfavorable clinical parameters of CRC. The observed association between HSP90 and Topo I in respect to worse clinical parameters reflects that cooperation between these molecules implies higher growth aggressiveness in the subset of HSP90/Topo I positive CRCs.

In summary, our results revealed that cooperation between HSP90 and Topo I expression exists in CRCs, independently of KRAS gene status, suggesting that synergistic effects of these molecules might promote higher invasiveness of HSP90/Topo I-positive tumors.

Parallel HSP90/Topo I expression in tumor cells could be potential target for the development of novel therapies for KRAS-mutated CRC.

\section{Conflicts of Interest}

The Authors declare that there are no conflicts of interest.

\section{Funding}

This study was supported by grant No 870 from Medical University Wroclaw, Poland.

\section{References}

1 Palomba G, Cossu A, Paliogiannis P, Pazzola A, Baldino G, Scartozzi M, Ionta MF, Ortu S, Capelli F, Lanzilo A, Sedda T, Sanna G, Barca M, Virdis L, Budroni M and Palmeieri G: Prognostic role of KRAS mutations in Sardinian patients with colorectal carcinoma. Oncol Lett 12: 1415-1421, 2016. 
2 Gilbert DC, Chalmers AJ and El-Khamisy SF: Topoisomerase I inhibition in colorectal cancer: biomarkers and therapeutic targets. Br J Cancer 108: 18-24, 2012.

3 Bruera G, Cannita K, Di Giacomo D, Lamy A, Frebourg Th, Sabourin JC, Tosi M, Alesse E, Ficorella C and Ricevuto E: Worse prognosis of KRAS c. $35 \mathrm{G}>\mathrm{A}$ mutant metastatic colorectal cancer (MCRC) patients treated with intensive triple chemotherapy plus bevacizumab (Flr-B/Fox). BMC Med 11: 59-69, 2013.

4 Hsu H-C, Thiam TK, Lu Y-J, Yeh CY, Tsai W-S, You JF, Hung HY, Tsai C-N, Hsu A, Chen H-C, Chen S-J and Yang T-S: Mutations of KRAS/NRAS/BRAF predict cetuximab resistance in metastatic colorectal cancer patients. Oncotarget 7: 2225722270, 2016.

5 Wang J, Cui S, Zhang X, Wu Y and Tang H: High Expression of heat shock protein 90 is associated with tumor aggressiveness and poor prognosis in patients with advanced gastric cancer. PLoS ONE 6: e62876, 2013.

6 Drecoll E, Nitche U, Bauer K, Berezowska S, Slotta-Huspenina $\mathrm{J}$, Rossenberg $\mathrm{R}$ and Langer R: Expression analysis of heat shock protein 90 (HSP90) and Her2 in colon carcinoma. Int $\mathrm{J}$ Colorectal Dis 29: 663-671, 2014.

7 Cercek A, Shia J, Gollub M, Chou JF, Capanu M, Raasch P, Reidy-Lagunes D, Proia DA, Vakiani E, Solit DB and Saltz L: Ganetespib: A novel Hsp90 inhibitor in patients with KRAS mutated and wild type, refractory metastatic colorectal cancer. Clin Colorectal Cancer 4: 207-212, 2014.

8 Phipps AI, Buchanan DD, Makar KW, Win AK, Baron JA, Lindor NM, Potter JD and Newcomb PA: KRAS-mutation status in relation to colorectal cancer survival: the joint impact of correlated tumour markers. Br J Cancer 108: 1757-1764, 2013.

9 Sasaki Y, Akasu T, Saito N, Kojima H, Matsuda K, Nakamori S, Komori K, Amagai K, Yamaguchi T, Ohue M, Nagashima K and Yamada Y: Prognostic and predictive value of extended RAS mutation and mismatch repair status in stage III colorectal cancer. Cancer Sci 7: 1006-1012, 2016.

10 Li Z-Z, Bai L, Wang F, Zhang Z-C, Wang F, Zeng Z-L, Zeng JB, Zhang D-S, Wang F-H, Wang Z-Q, Li Y-H, Shao J-Y and Xu $\mathrm{R}-\mathrm{H}$ : Comparison of KRAS mutation status between primary tumor and metastasis in Chinese colorectal cancer patients. Med Oncol 33: 33-71, 2016.

11 Uemura M, Kim HM, Hata T, Sakata K, Okuyama M, Takemoto H, Fujii H, Fukuzaki T, Morita T, Hata T, Takemasa I, Satoh T, Mizushima T, Doki Y and Mori M: First-line cetuximab-based chemotherapies for patients with advanced or metastatic KRAS wild-type colorectal cancer. Mol Clin Oncol 5: 375-379, 2016.

12 Meisenberg C, Gilbert DC, Chaimers A, Haley V, Gollins S, Ward SE and El-Khamisy SF: Clinical and cellular roles for TDP1 and TOP1 in modulating colorectal cancer response to irinotecan. Mol Cancer Ther 14: 575-585, 2015.

13 Seo YH: Dual inhibitors against topoisomerases and histone deacetylases. J Cancer Prev 20: 85-91, 2015.

$14 \mathrm{Lu} \mathrm{B}$, Zhang H, Zhang T, Cai Y, Hu Y, Zheng H and Li B: Topoisomerase I expression is associated with prognosis in postoperative non-small cell lung cancer patients. Thorac Cancer 7: 486-494, 2016.

15 Alagoz M, Gilbert DC, El-Khamisy S and Chaimers AJ: DNA repair and resitance to topoismerase I inhibitors: mechanisms, biomarkers and therapeutic targets. Curr Med Chem 19: 3874$3885,2012$.
16 Xu Q-R, Liu X, Yao Y-M and Liu Q-G: Expression of HSP90 and HIF-1, in human colorectal cancer tissue and its significance. Asian Pac J Trop Med 7: 720-724, 2014.

17 Tatokoro M, Koga F, Yoshida S and Kihara K: Heat shock protein 90 targeting therapy: state of the art and future perspective. EXCLI J 14: 48-58, 2015.

18 Wang M, Shen A, Zhang C, Song Z, Ai J, Liu H, Sun L, Ding J, Geng $M$ and Zhang A: Development of heat shock protein (Hsp90) inhibitors to combat resistance to tyrosine kinase inhibitors through Hsp90-kinase interactions. J Med Chem 59: 5563-5586, 2016.

19 Pillai RN and Ramalingam SS: Heat shock protein 90 inhibitors in non-small-cell lung cancer. Curr Opin Oncol 2: 159-164, 2014.

20 Cao Z-W, Zeng Q, Pei H-J Ren L-D, Bai H-Z and Na R-N: HSP90 expression and its association with wighteone metabolite response in HER2-positive breast cancer cells. Oncol Lett 11: 3719-3722, 2016.

21 Ahn J-Y, Lee J-S, Min H-Y and Lee H-Y: Acquired resistance to 5-fluorouracil via HSP90/Src-mediated increase in thymidylate synthase expression in colon cancer. Oncotarget 32 : 32622-32633, 2015.

22 Hamilton SR, Bosman FT, Boffetta P, Ilyas M and Morreau H: Carcinoma of colon and rectum. In: WHO Classification of Tumours of the Digestive System. Bosman FT, Carneiro F, Hruban RH, Theise ND, eds. Lyon: IARC Press, p. 131-182, 2010.

23 Azzoni C, Bottarelli L, Cecchini S, Ziccarelli A, Campanini N, Bordi C, Sarli L and Silini EM: Role of topoisomerase I and thymidylate synthase expression in sporadic colorectal cancer: Association with clinicopathological and molecular features. Pathol Res Pract 210: 113-117, 2014.

24 Koumarianou A, Tzeveleki I, Mekras D, Eleftheraki AG, Bobos M, Wirtz R, Fountzilas E, Valavanis C, Xanthakis I, Kalogeras KT, Basdanis G, Pentheroudakis G, Kotoula V and Fountzilas G: Prognostic markers in early-stage colorectal cancer: significance of TYMS mRNA expression. Anticancer Res 34: 4949-4962, 2014.

25 Nygard SB, Vainer B, Nielsen SL, Bosman F, Tejpar S, Roth A, Delorenzi $\mathrm{M}$, Brùnner $\mathrm{N}$ and Budinska $\mathrm{E}$ : DNA topoisomerase I gene copy number and mRNA assessed as predictive biomarkers for adjuvant irinotecan in stage II/III colon cancer. Clin Cancer Res 22: 1621-1631, 2016.

26 Ghidini M, Personeni N, Bozzarelli S, Baretti M, Basso G, Bianchi P, Tronconi MC, Pressiani T, Grizzi F, Giordano L, Malesci A, Alloisio M, Laghi L, Santoro A and Rimassa L: KRAS mutation in lung metastases from colorectal cancer: prognostic implications. Cancer Med 5: 256-264, 2016.

27 Watson R, Liu T-Cand Ruzinova MB: High frequency of KRAS mutation in early onset colorectal adenocarcinoma: implications for pathogenesis. Hum Pathol 56: 163-170, 2016. 